\title{
An Enhanced Impulsive Noise Suppression Method Based on Wavelet Denoising and ICA for Power Line Communication
}

\author{
Wei Zhang, Zhongqiang Luo, Xingzhong Xiong, and Kai Deng
}

\begin{abstract}
Aiming at the problem of noise suppression in power lines, traditional noise suppression methods need to know prior knowledge and other defects. In this paper, blind source separation methods that do not need prior knowledge are selected. In the case of low signal-to-noise ratio, the basic independent component analysis algorithm has poor denoising effect. Therefore, this paper proposes a joint independent component analysis algorithm based on Wavelet denoising and Power independent component analysis (WD-PowerICA). In this work, firstly, the pseudo observation signal is constructed by weighted processing, and the blind separation model of single channel is transformed into a multi-channel determined model. Then, the proposed WD-PowerICA algorithm is used to separate noise and source signals. Finally, the simulation results demonstrate that the proposed algorithm in this paper can effectively separate noise and source signal under low SNR. At the same time, the stronger the $\alpha$ pulse noise is, the closer the WD-PowerICA separated signal is to the source signal. The proposed algorithm is better than the state of the art PowerICA algorithm.
\end{abstract}

Index Terms-Impulse noise, Blind source separation, Power line communication, Independent component analysis, Orthogonal frequency division multiplexing.

\section{INTRODUCTION}

$\mathrm{P}_{\mathrm{p}}^{\mathrm{om}}$ OWER grid is the largest, most popular and most reliable power supply carrier. Power line communication(PLC) technology is a new technology that utilizes widely existing power lines for communication and has received increasing attention [1]. The research on power line carrier communication technology is mainly carried out from three aspects, namely signal attenuation characteristics, impedance characteristics and noise characteristics [2-3]. Among them, noise interference is one of the most important factors affecting the success rate of power line carrier communication. The noise in power line carrier communication is far more complicated than that in other dedicated communication lines. It is found that power line noise can be divided into five types of noise:

This work was supported by National Natural Science Foundations of China (No. 61201266 ), Scientific research projects of Yibin University (No. 2021PY20)

Wei Zhang and Kai Deng are with Yibin University, Yibin 644000, China (Corresponding author: 991728174@qq.com).

Zhongqiang Luo and Xingzhong Xiong are with Artificial Intelligence Key Laboratory of Sichuan Province, Sichuan University of Science and Engineering, Yibin 644000, China. colored background noise, narrowband noise, periodic impulse noise asynchronous to power frequency, and period synchronized with power frequency, impulse noise and asynchronous impulse noise. Most of the literature classifies the first three as background noise, the latter two being called impulse noise. Impulse noise is the main detrimental cause of affecting communication quality [4]. Therefore, it is necessary to take effective measures to solve impulse noise for power line stable and reliable communication.

So far, scholars around the world have done a lot of research on noise removal of PLC systems based on OFDM signals. In [5-6] provide time-domain elimination methods for impulse noise in PLC, including limiting, zeroing, and a combination of the two. These methods are so simple and can also improve the performance of the system to a certain extent. However, the optimal threshold calculation of these algorithms is very difficult. Therefore, in practice, it is often set by experience, which not only limits the performance of this type of method but also destroys the orthogonality between OFDM subcarriers [7]. To solve the problem of inter-carrier interference caused by blanking, an iterative interference cancellation method is proposed [8], but this method has a slower convergence rate. A method for eliminating impulse noise combined with equalization in the frequency domain is proposed [9], the method reconstructs noise by estimating the time domain position, amplitude and phase of the occurrence of impulse noise, and the implementation process is very complicated. The compressive sensing method needs to meet the following constraints: the number of impulse signals in an OFDM symbol cannot exceed the minimum threshold of the number of Fourier transform points and the number of empty subcarriers [10], due to the random of the impulse noise signal itself, the above constraints limit the use of this method. In [11], based on the sparse Bayesian learning method, according to the decision regression detection, the impulse noise signal is reconstructed and then eliminated. However, when reconstructing the impulse noise signal, it is necessary to know the state information of impulse noise and Gaussian noise, and the calculation complexity is high, which is difficult to deal with in the actual system. In [12], a fractional low order independent component analysis algorithm based on negative entropy is proposed to remove mixed noise. This algorithm can protect the pure signal in the mixed signal, and does not need the characteristic parameter of noise. However, the separation 
effect of this algorithm is terrible, and it is not good at low signal-to-noise ratio(SNR).

Through the research analysis of previous scholars, we know that some existing methods have certain limitations. Due to the complexity and variability of power channels, the advanced algorithm of blind source separation, i.e. power iterative independent component analysis algorithm(PowerICA), is still selected in this project, which can achieve fast and stable separation of mixed signals [13]. But this algorithm is not ideal at low SNR. Therefore, this study combines wavelet denoising and power iterative independent component analysis algorithm to denoise (WD-PowerICA). The simulation results show that the de-noising effect is very good. At the same time, it makes up for the disadvantage of power iteration algorithm in low SNR denoising performance, so that we can receive the required signals stably and reliably.

The rest of the paper is structured as follows: In section II, we construct the model of the PLC system and the model of signal as well as problem formulation; In section III, the basic principles of FastICA, PowerICA, WD-PowerICA are illustrated; The simulation results and brief analysis are presented in Section IV; Section V summarizes the experimental results and gives the next research ideas.

\section{Signal Model AND Problem Formulation}

Random impulse noise is mainly caused by the switch of household appliances and lightning in natural phenomena. This kind of noise is characterized by strong randomness, short duration, large energy, uncertain pulse interval and pulse width, and serious interference to power line communication. Some researchers model the noise of PLC as Middleton Class A model [14]. Literature [15] points out that Middleton Class A model cannot accurately describe the noise in PLC channels. Through the actual measurement of the background noise and pulse noise of PLC system, a noise model that obeys $\alpha$ stable distribution is proposed [16]. The actual measured values in reference [17] show that as a special case of $\alpha$ stable distribution, symmetric alpha stable $(\mathrm{S} \alpha \mathrm{S})$ distribution not only includes the case of Gaussian distribution (i.e. characteristic factor $\alpha=2$ ), but also can reflect the pulse characteristics of background noise, which is full of generalized central limit theorem [18]. The probability density function of $\alpha$ stable distribution has no uniform expression, and is generally described by its characteristic function. In [19], it can be expressed as follow:

$$
\varphi(t)=\exp \left(j p t-\gamma|t|^{\alpha}[1+j \beta s g n(t) \omega(t, \alpha)]\right)
$$

Where

$$
\omega(t, \alpha)=\left\{\begin{array}{l}
\tan \frac{\alpha \pi}{2}, \alpha \neq 1 \\
\frac{2}{\pi} \log |t|, \alpha=1
\end{array}\right.
$$

Among them $\alpha \in(0,2]$, is the characteristic index, which determines the degree of pulse characteristics of the distribution. The smaller the $\alpha$ value is, the more significant the pulse characteristics are. When $\alpha=2$, the characteristic function formula is the same as that of the Gaussian distribution with the mean value of a and variance of $2 \delta^{2}$, that is, the Gaussian distribution is a special case of the $\alpha$ stable distribution; $-1<\beta<1$ is the symmetric parameter, $\beta=0$ is the symmetric distribution; $\gamma$ is the dispersion coefficient, which is a measure of the dispersion degree of samples relative to the mean value, similar to the variance in the Gaussian distribution; $p \in(-\infty,+\infty)$ is the position parameter. For $\mathrm{S} \alpha \mathrm{S}$ distribution, if $0<\alpha \leq 1, p$ represents the median value; if $1<\alpha \leq 2, p$ is the mean value.

The indoor single phase power line consists of three wires: phase wire $(\mathrm{P})$, neutral wire $(\mathrm{N})$, protection earth wire $(\mathrm{PE})$. It can provide multiple feeding and receiving ports for the communication system: P-N, P-PE, and N-PE. The voltage difference between two power lines can be called a port, like an antenna in a wireless network, these PLC ports can be used as communication ports for signal transmission and reception, but they need to meet Kirchhoff's rule. Therefore, the transmitter can only use two antennas [20], the model of PLC system is shown in Figure 1.

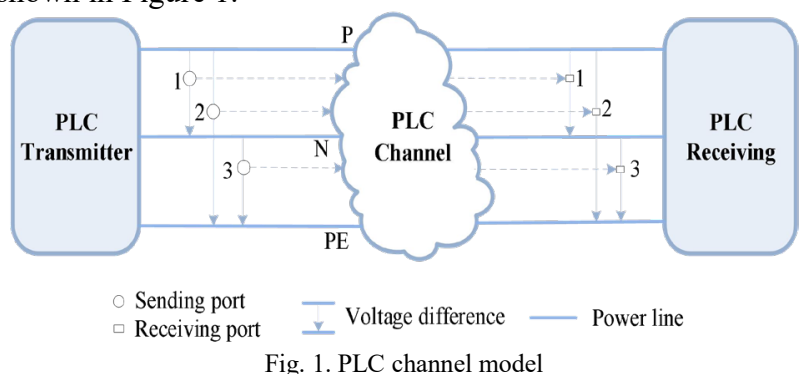

In the process of signal transmission, the channel model of the system is equipped with 2 feeding ports and 2 receiving ports $(2 \times 2)$. Assume that both Gaussian noise and impulse noise exist at the same time. The noise model of receiving mixed signal can be expressed as follows:

$$
\boldsymbol{X}=\left[\begin{array}{ll}
a_{11} & a_{12} \\
a_{21} & a_{22}
\end{array}\right]\left[\begin{array}{l}
\boldsymbol{s} \\
\boldsymbol{v}
\end{array}\right]+\boldsymbol{n}
$$

where $\boldsymbol{X}$ is an observation signal, $\boldsymbol{s}$ is OFDM source signal, $\boldsymbol{v}$ is impulse noise subject to stable distribution, $\boldsymbol{n}$ is Gaussian noise, $a_{i j}$ is equivalent to complex channel influence factor. $\boldsymbol{s}, \boldsymbol{v}$ are the system input signal, $\boldsymbol{n}$ is PLC channel random generation. Due to the advantages of blind separation [21], we don't need to estimate the channel influence factor and synchronization parameter. It is significant to use the blind adaptive separation algorithm to achieve noise cancellation in low SNR.

\section{BLIND SEPARATION Algorithm BASED ON WD-POWERICA}

BSS is a kind of adaptive signal processing, which is used to retrieve multi-channel mixed original signals transmitted from multiple point sources. Because the source signal and the mixed channel are unknown, it is called blind source separation. The task of BSS is to recover the original sources from their linear 
instantaneous mixing only dependent on the statistical independent sources. The ICA analysis is the main method to solve the problem of blind source separation. Its properties depend on the independence criterion and optimization algorithm. Non-Gaussian is a common criterion for ICA. According to the generalized central limit theorem, the non-Gaussian criterion can be used as the cost function to maximize the non-Gaussian to achieve the purpose of extracting independent sources. The ICA linear mixed model can be expressed as [13]:

$$
\boldsymbol{X}=\boldsymbol{A S}+\boldsymbol{n}
$$

Where $\boldsymbol{X}=\left[\begin{array}{llll}x_{1} & x_{2} & \cdots & x_{M}\end{array}\right]^{\mathrm{T}} \quad$ represents observation signal, $\boldsymbol{A}$ is a $M \times N$ unknowing mixed matrix, $\boldsymbol{S}=\left[\begin{array}{llll}s_{1} & s_{2} & \cdots & s_{N}\end{array}\right]^{\mathrm{T}}$ represents the system input signal. According to the ICA principle, The model of received noise signal in this paper is shown in equation (3), the noise signal blind source separation model is shown in figure 2 .

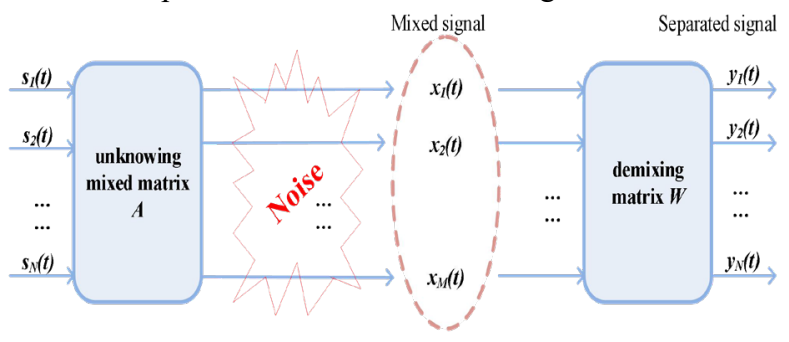

Fig. 2. Noisy blind source separation model

\section{A. FastICA Algorithm}

Fast independent component analysis(FastICA) is an iterative estimation method based on information theory and principle. It is an estimation algorithm based on negative entropy maximization, negentropy is obtained from differential entropy and defined as [22]:

$$
J(\boldsymbol{Y})=H\left(\boldsymbol{Y}_{\text {Gauss }}\right)-H(\boldsymbol{Y})
$$

where $\boldsymbol{Y}_{\text {Gauss }}$ and $\boldsymbol{Y}$ are the random variables with the same covariance And the expression of the differential entropy $H(\boldsymbol{Y})$ :

$$
H(\boldsymbol{Y})=-\int P_{Y}(\xi) \log P_{Y}(\xi) d \xi
$$

FastICA is an iterative calculation process. The rule it learns is to explore the direction which gains the maximum degree of non-Gaussian in terms of the equation . The FastICA algorithm finds the demixing matrix by iteration objective function:

$$
J(\boldsymbol{y}) \approx c[E\{G(\boldsymbol{y})\}-E\{G(\boldsymbol{v})\}]^{2}
$$

$c$ is a positive constant and $v$ is a random variable of the Gaussian with zero mean and unit variance, $G$ is a non-quadratic function. Since OFDM signal is sub-Gaussian, $G$ can be expressed as:

$$
G(\boldsymbol{u})=\frac{1}{a} \log _{2} \cosh (a \boldsymbol{u}), G^{\prime}(\boldsymbol{u})=\tanh (a \boldsymbol{u})
$$

Where $a \in[1,2]$.
Due to $\boldsymbol{y}=\boldsymbol{w}^{\mathrm{T}} \boldsymbol{X}$, equation (7) can be written as:

$$
J_{G}(\boldsymbol{W}) \propto\left\{E\left[G\left(\boldsymbol{w}^{T} \boldsymbol{X}\right)\right]-E[G(\boldsymbol{v})]\right\}^{2}
$$

The maximum $J_{G}(\boldsymbol{W})$ is converted to finding the maximum value of the separation matrix $\boldsymbol{W}$. FastICA estimator maximizes the Lagrangian.

$$
\mathcal{L}(\boldsymbol{w} ; \lambda)=\left|\mathbb{E}\left[G\left(\boldsymbol{w}^{T} \boldsymbol{X}\right)\right]\right|-\frac{\lambda}{2}\left(\boldsymbol{w}^{T} \boldsymbol{w}-1\right)
$$

Here, $\lambda$ is the Lagrange multiplier. Due to the local optimum of (10), the following equations hold

$$
F(\boldsymbol{w})=\mathrm{m}(\boldsymbol{w})-\lambda(\boldsymbol{w}) \boldsymbol{w}=0
$$

where $\mathrm{m}(\boldsymbol{w})=\mathbb{E}\left[g\left(\boldsymbol{w}^{\mathrm{T}} \boldsymbol{X}\right) \boldsymbol{X}\right]$ and $\lambda(\boldsymbol{w})=\boldsymbol{w}^{T} m$ can be obtained by multiplying $w^{T}$ from the left on both sides of equation (11), $\lambda(\boldsymbol{w})$ is treated as a constant that does not depend on $w$. the one-unit fixed point FastICA algorithm is used as an approximate Newton-Raphson iterative update. The iteration of FastICA can be further expressed as:

$$
\boldsymbol{w} \leftarrow \frac{\mathrm{m}(\boldsymbol{w})-\beta(\boldsymbol{w}) \boldsymbol{w}}{\|\mathrm{m}(\boldsymbol{w})-\beta(\boldsymbol{w}) \boldsymbol{w}\|}
$$

until convergence, $\quad \beta(\boldsymbol{w})=\mathbb{E}\left[g^{\prime}\left(\boldsymbol{w}^{\mathrm{T}} \boldsymbol{X}\right)\right] \in \mathbb{R}$ is a scalar multiplier, $g=G^{\prime}$.

\section{B. WD-PowerICA Algorithm}

Use either SI (MKS) or CGS as primary units. (SI units are strongly encouraged.) English units may be used as secondary units (in parentheses). This applies to papers in data storage. For example, write " $15 \mathrm{~Gb} / \mathrm{cm}^{2}\left(100 \mathrm{~Gb} / \mathrm{in}^{2}\right)$." An exception is when English units are used as identifiers in trade, such as " $3 \frac{1}{2} 2$-in disk drive." Avoid combining SI and CGS units, such as current in amperes and magnetic field in oersteds. This often leads to confusion because equations do not balance dimensionally. If you must use mixed units, clearly state the units for each quantity in an equation.

Wavelet analysis consists of breaking up a signal into scaled and shifted versions of the original signal or mother wavelet. Wavelets are family of functions constructed from translations and dilations of a single function, they are defined by:

$$
\psi_{a, b}(t)=\frac{1}{\sqrt{a}} \psi\left(\frac{t-b}{a}\right), a \neq 0
$$

Where $\psi_{a, b}(t)$ is called daughter wavelet, $\psi(t)$ is called mother wavelet, the parameter $a$ is the scaling parameter or scale, and it measures the degree of compression, the parameter $b$ is the shift parameter which determines the time location of the wavelet [23].

Shahab Basiri et al. propose a novel power iteration algorithm for FastICA, which is numerically more stable than the original FastICA algorithm, when the sample size is not orders of magnitudes larger than the dimension [24]. The algorithm does not require using unnecessary assumptions, the Lagrangian multiplier is not treated as a constant and an ad-hoc approximation is not used for Jacobian matrix in the NR update, the method can be run on parallel computing nodes, which 
drastically reduces the computational time. Therefore, FastICA as a further expression of the PI method:

$$
\boldsymbol{w} \leftarrow \frac{[\mathrm{H}(\boldsymbol{w})-\beta(\boldsymbol{w}) \mathrm{I}] \boldsymbol{w}}{\|[\mathrm{H}(\boldsymbol{w})-\beta(\boldsymbol{w}) \mathrm{I}] \boldsymbol{w}\|}
$$

Where $\mathrm{H}(\boldsymbol{w})=\mathbb{E}\left[\frac{g\left(\boldsymbol{w}^{\mathrm{T}} \boldsymbol{X}\right)}{\boldsymbol{w}^{\mathrm{T}} \boldsymbol{X}} \boldsymbol{X} \boldsymbol{X}^{\mathrm{T}}\right] \in \mathbb{R}^{d \times d}$ is positive definite for all conventional ICA nonlinearities.

Following the previous work [13], this article studies the separation effect of the PowerICA algorithm under low signal-to-noise ratio conditions. Simulation experiments verify that the separation effect is not ideal. Therefore, under the condition of low SNR, this time the wavelet denoising is used to preprocess the mixed signal, the signals $\boldsymbol{X}^{\prime}(t)$ processed by wavelet transform is:

$$
\begin{aligned}
\boldsymbol{X}^{\prime}(t)=\left\langle\boldsymbol{X}(t), \psi_{a, b}(t)\right\rangle & =\int_{-\infty}^{\infty} \boldsymbol{X}(t) \psi_{a, b}^{*}(t) d t \\
& =\int_{-\infty}^{\infty}(\boldsymbol{A} \boldsymbol{S}(t)+\boldsymbol{N}(t)) \psi_{a, b}^{*}(t) d t
\end{aligned}
$$

After preprocessing the mixed signals using wavelet denoising and then it is separated by PowerICA. Experimental simulations verify that the combination of the two algorithms(ie, WD-PowerICA) can achieve stable and efficient separation [25]. The specific steps are:

1. After receiving the entire observation signal, remove the cyclic prefix from the OFDM signal, and then perform the fast Fourier transform.

2. Apply wavelet denoising to preprocess the observation signals with random weights.

3. Perform PowerICA processing on the $\mathrm{N}$ sub-carriers to estimate the MIMO channel of the N sub-carriers.

4. Use interpolation to obtain channel estimates for the remaining subcarriers.

The processing flow chart is illustrated in Figure 3.

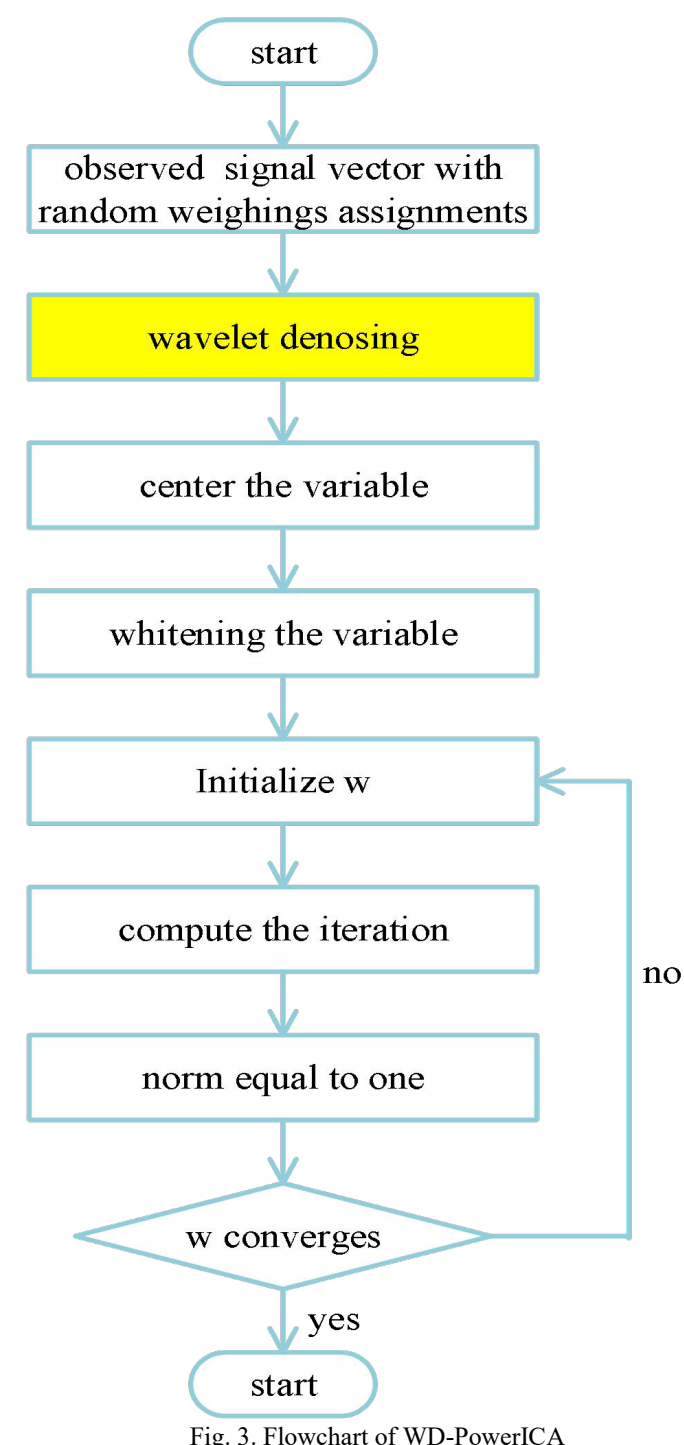

Next, the computational cost of the WD-PowerICA Algorithm will be analyzed simply, $\boldsymbol{X}=\boldsymbol{A} \boldsymbol{S}+\boldsymbol{n}$ takes $O(M N)$ operations. Therefore, the computational cost is described as,

$$
O(\text { total })=\text { iterations } \times(O(M N))
$$

From above analysis, although the computational steps of the proposed algorithm are relatively complex, the computational complexity remains unchanged. However, the WD-PowerICA algorithm is very important to improve the performance of power line communication in the case of low SNR.

\section{Simulation ANALYSiS AND Discussion}

To demonstrate the effectiveness of the proposed WD-PowerICA algorithm based blind source separation for power line communication system at low SNR, we conduct simulation experiments to evaluate the performance. In the experiment, the signal noise model is shown in section II. The useful signal $s$ is the OFDM signal, and $v$ is the impulse noise, they are seen as two input source signals, the carrier frequency is 1000 and sample frequency is 2000 , and the SNR of input 
signal is $8 \mathrm{~dB}$, and the number of sample points is 500 when $\alpha=0.8, \beta=0, \gamma=1, \lambda=0$, the original input signals are shown in Figure 4.
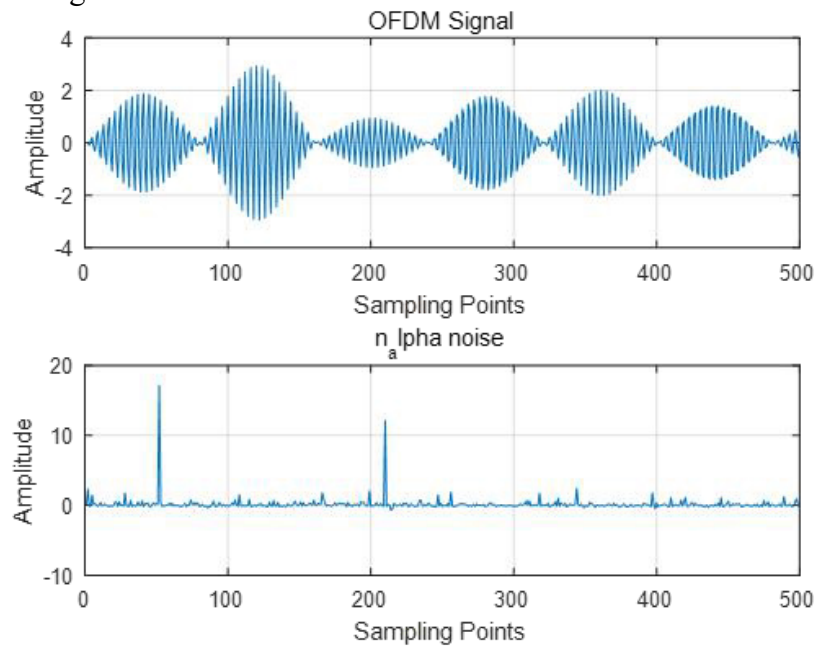

Fig. 4. The original input signals

To generate the two random mixed observation signals, the two random number couples used as mixing weighing assignment vectors are multiplied respectively with the original input signals, and the observation signals are shown Figure 5.
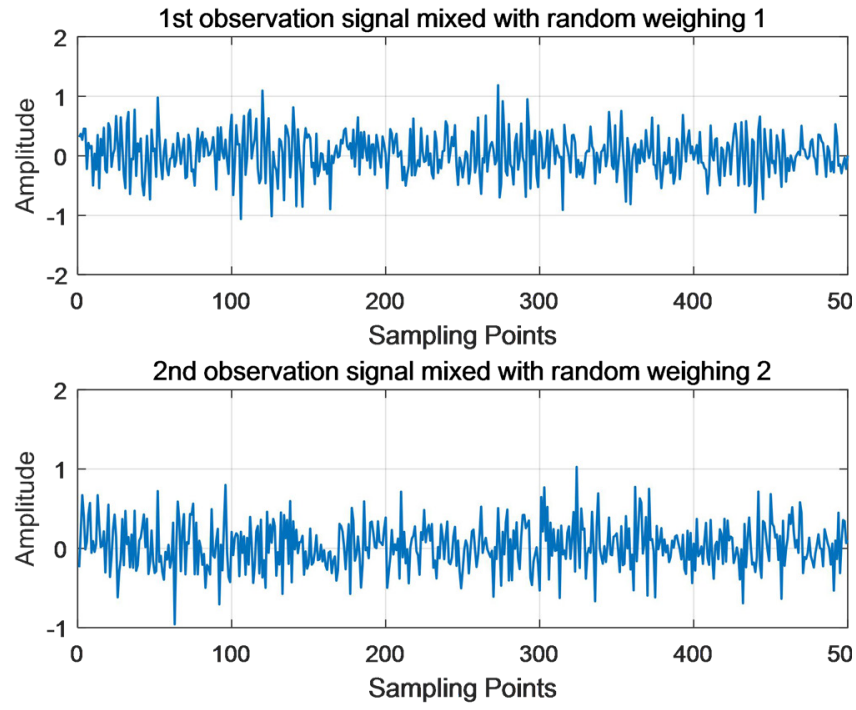

Fig. 5. Observation signals

ICA algorithm is based on the statistical characteristics of signals to maximize the independence between signals, so as to achieve the purpose of estimating the source signal. Compared with Gaussian white noise and OFDM signals, the $\alpha$-stable distribution noise is the strongest. Therefore, the impulse noise can be extracted first. By FastICA, PowerICA and WD-PowerICA, the separation results are shown in (a), (b) and (c) of Figure 6 .
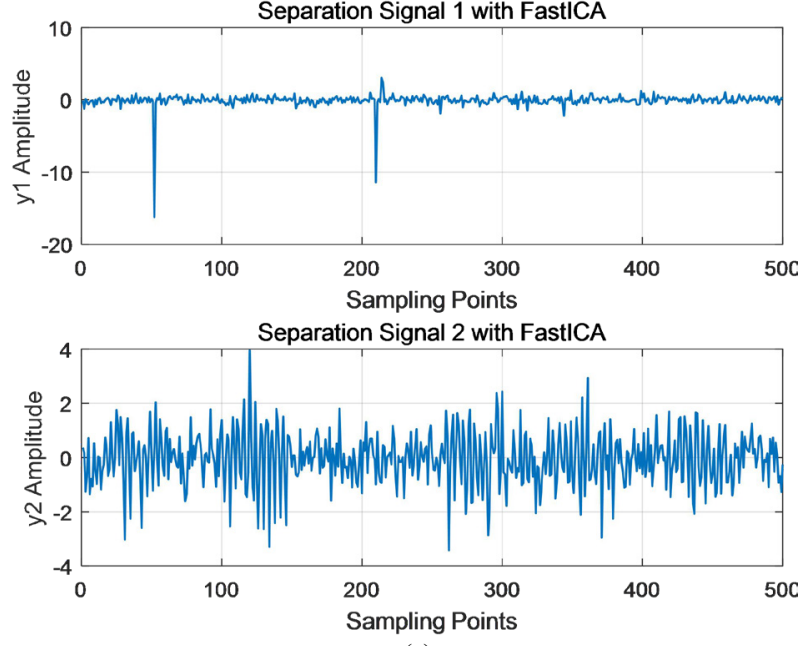

(a)
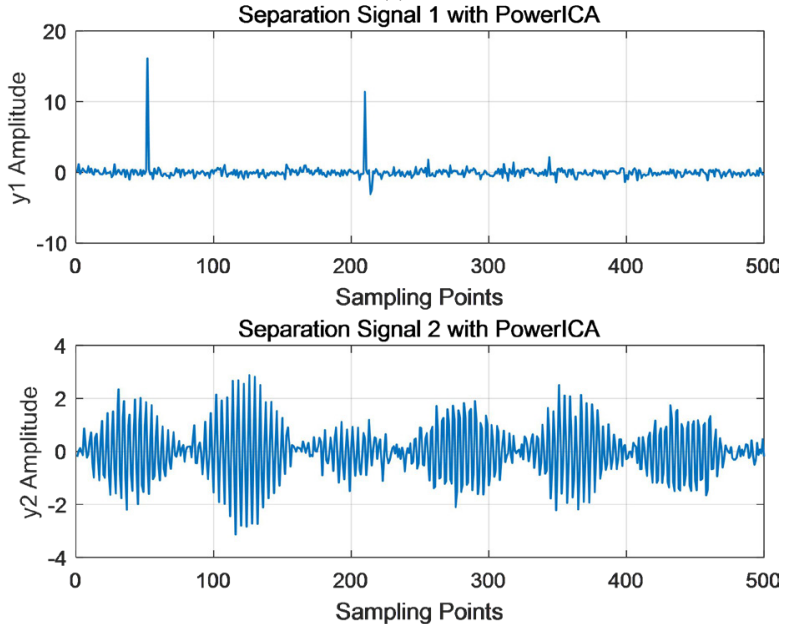

(b)
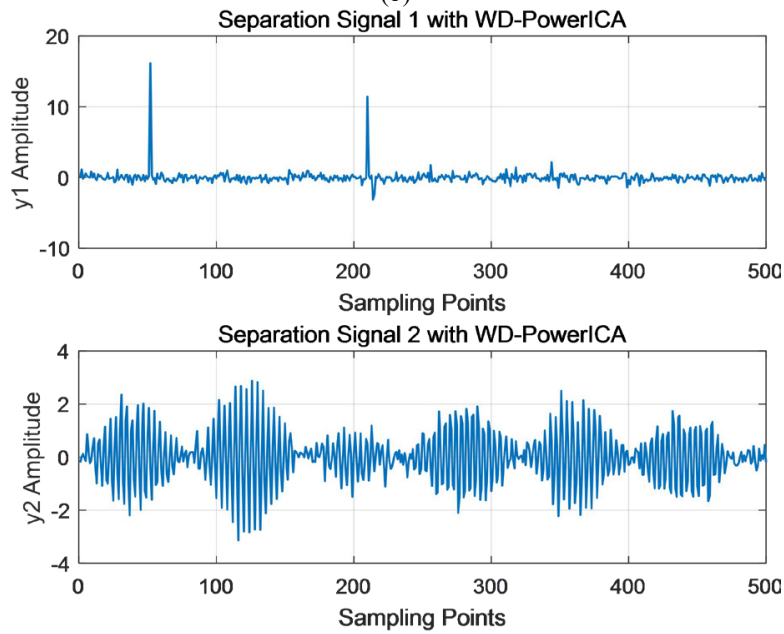

(c)

Fig. 6. Algorithm separation result: (a) FastICA algorithm separation; (b) PowerICA algorithm separation; (c) WD-PowerICA algorithm separation.

In the simulation experiment, other conditions remain unchanged, only the SNR is changed, and the separation effect of the three algorithms is shown in Figure 7. Through the experimental simulation, the algorithm in this paper is 
obviously superior to the other two algorithms, especially in the case of low signal-to-noise ratio.

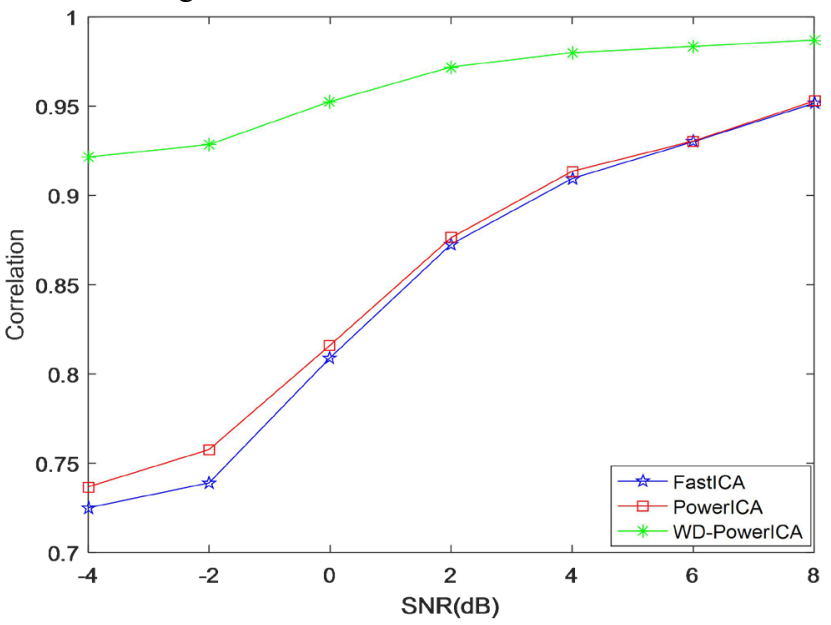

Fig. 7. Separation result of changing SNR

Due to $\alpha \in(0,2]$, the larger $\alpha$, the stronger Gauss, changing the value describing the Gaussian strength of impulse noise, the experimental results are as shown in Figure 8. When the input signal is very non-Gaussian, the algorithm in this paper has a better separation effect. However, When $\alpha>1.1$ approximately, WD-PowerICA algorithm performance is significantly reduced, This is my next problem to be solved.

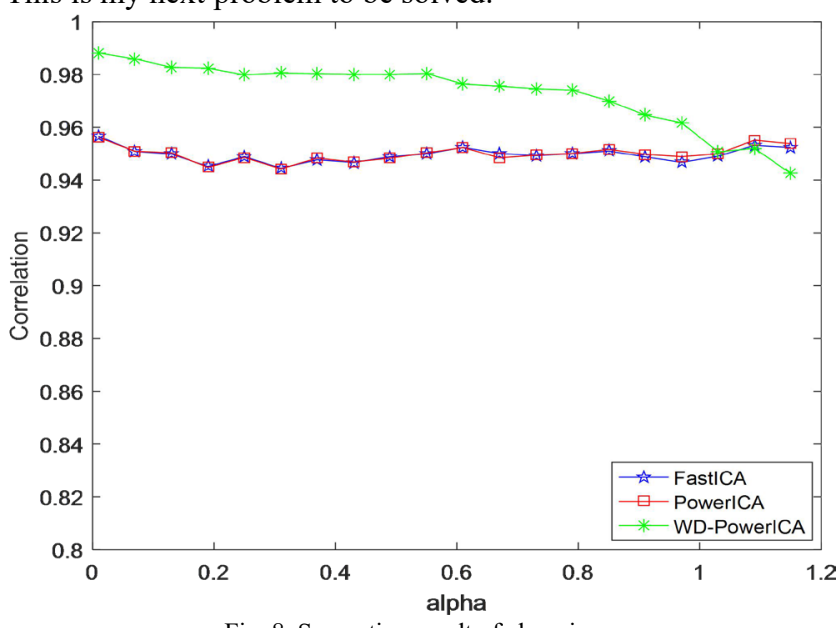

Fig. 8. Separation result of changing

\section{CONCLUSIONS}

This paper studies the denoising method of power line communication in low signal-to-noise ratio. Aiming at the situation that the denoising effect of PowerICA algorithm is not ideal in low signal-to-noise ratio, combined with the advantages of wavelet denoising algorithm, a joint denoising method combining wavelet and PowerICA algorithm is proposed. The WD-PowerICA denoising effect is obviously improved, and the effective separation of noise and useful signal is realized. However, due to the limited time, this paper only analyzes the correlation index. Next, we will study the BER and other performance indicators.

\section{REFERENCES}

[1] Baig S., Asif H. M., Umer T., et al.: 'High data rate discrete wavelet transform-based PLC-VLC design for 5G communication systems'. IEEE Access, 2018, 6, pp. 52490-52499.

[2] Songnong L., Xiaorui H., Ke Z., et al.: 'Measurement and research on attenuation characteristics of low voltage power line communication channel'. Power Line System Protection and Control, 2018, 46, (4), pp. 99-106.

[3] Ying C., Xiaosheng L., Dianguo X.: 'Game analysis and optimization of communication network performance for low voltage power line'. Automation of Electric Power Systems, 2018, 42, (11), pp. 122-128. DoI: $10.7500 /$ AEPS20170807005.

[4] Hui Z., Wenbing L., Xiongwen Z., et al.: 'Noise modeling for power line communication channel using the LS-SVM and wavelet neural networks'. Transactions of China Electrotechnical Society, 2018, 33, (16), pp. 3879-3888.

[5] Juwono F. H., Guo Q., Huang D., et al.: 'Deep clipping for impulsive noise mitigation in OFDM-based power-line communications'. IEEE Transactions on Power Delivery, 2014, 29, (3), pp. 1335-1343.

[6] Papilaya V .N., Vinck A.J.H.: 'Investigation on a new combined impulsive noise mitigation scheme for OFDM transmission'. International Symposium on Power Line Communications and Its Applications, Johannesburg, South Africa, March, 2013, pp. 86-91.

[7] Darsena D., Gelli G., Melito F., et al.: 'ICI-free equalization in OFDM systems with blanking preprocessing at the receiver for impulsive noise mitigation'. IEEE Signal Processing Letters, 2015, 22, (9), pp. 1321-1325.

[8] Yih C. H.: 'Iterative interference cancellation for OFDM signals with blanking nonlinearity in impulsive noise channels'. IEEE Signal Processing Letters, 2012, 19, (3), pp. 147-150.

[9] Ando H., Nakamura A., Ohno K., et al.: 'A study on channel estimation under class A impulsive PLC channel'. International Symposium on Power Line Communications and Its Applications, Johannesburg, South Africa, March, 2013, pp. 69-70.

[10] Mehboob A., Li Z., Khangosstar J.: 'Adaptive impulsive noise mitigation using multi mode compressive sensing for powerline communications'. International Symposium on Power Line Communications and Its Applications, Beijing, China, March, 2012, pp. 368-373.

[11] Lin J., Nassar M., Evans B. L.: 'Impulsive noise mitigation in powerline communications using sparse Bayesian learing'. IEEE Journal on Selected Areas in Communications, 2013, 31, (7), pp. 1172-1183.

[12] Qiu T. S.,Li B., Zha D.F.: 'Elimination of pulse-noise from mixed-noise based on fractional lower order ICA'. Journal on Communications, 2011, 32, (9), pp. 77-81.

[13] Wei Z., Zhongqiang L., Xingzhong X.: 'Impulse Noise Suppression Based on Power Iterative ICA in Power Line Communication'. International Journal of Electronics and Telecommunications, 2019, 64, (4), pp. 651-656.

[14] Middleton D.: 'Statistical-physical models of electromagnetic interference'. IEEE Transactions on Electromagnetic Compatibility, 1977, EMC-19, (3), pp. 106-127. Dor: 10.1109/TEMC.1977.303527.

[15] Andreadou N., Pavlidou F. N.: 'Modeling the noise on the OFDM power-line communications system'. IEEE Transactions on Power Delivery, 2010, 25, (1), pp. 150-157. DOI: 10.1109/TPWRD.2009.2035295.

[16] Tran T. H., Do D. D., Huynh T. H.: 'PLC impulsive noise in industrial zone: Measurementand characterization'. International Journal of Computer and Electrical Engineering, 2013, 5, (1), pp. 48-51. DoI: 10.7763/IJCEE.2013.V5.660

[17] Laguna-Sanchez G., Lopez-Guerrero M.: 'On the use of alpha-stable distributions in noise modeling for PLC'. IEEE Transactions on Power Delivery, 2015, 30, (4), pp. 1863-1870. DoI: 10.1109/TPWRD.2015.2390134.

[18] Mahmood A., Chitre M.: 'Optimal and near-optimal detection in bursty impulsive noise'. IEEE Journal of Oceanic Engineering, 2017 42, (3), pp. 639-653. DoI: 10.1109/JOE.2016.2603790. 
An Enhanced Impulsive Noise Suppression Method Based on Wavelet Denoising and ICA for Power Line Communication

[19] Shao M., Nikias C. L.: 'Signal processing with fractional lower order moments: stable processes and their applications'. Proceedings of the IEEE, 1993, 81, (7), pp. 986-1010.

[20] Berger L. T., Schwager A., Pagani P., et al.: 'MIMO Power Line Communications: Narrow and Broadband Standards, EMC, and Advanced Processing'. (CRC Press, 2014, 1st edn.), pp.5-43.

[21] Zhongqiang L., Chengjie L., Lidong Z.: 'A Comprehensive Survey on Blind Source Separation for Wireless Adaptive Processing: Principles, Perspectives, Challenges and New Research Directions'. IEEE Access 2018, 6, pp. 66685-66708.

[22] Chengjie L., Lidong Z., Zhongqiang L.: 'Underdetermined Blind Source Separation of Adjacent Satellite Interference Based on Sparseness'. China Communications, 2017, 14, (2), pp. 140-149.

[23] Karanpreet Kaur. Disceret Wavelet Transform based OFDM System using Convolutional Encoding. Master thesis. Patiala: Thapar university; 2014, pp. 58-63.

[24] Basiri S., Ollila E., Koivunen V.: 'Alternative Derivation of FastICA With Novel Power Iteration Algorithm'. IEEE Signal Processing Letters, 2017, 24, (9), pp. 1378-1382.

[25] Abdel-Hamid G., Samir R.: 'Blind Channel Estimation Using Wavelet Denoising of Independent Component Analysis for LTE'. Indonesian Journal of Electrical Engineering and Computer Science, 2016, 17, (1), pp.126-137.

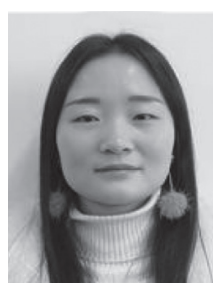

Wei Zhang received the B.S. and M.S. degrees in communication engineering and pattern recognition and intelligent systems from Sichuan University of Science and Engineering(SUSE), Zigong, China, in 2017 and 2020, respectively. Since Sep. 2020, she has been with Yibin University, Her research contain Blind Source Separation, intelligent Signal Processing and Artificial Intelligence.

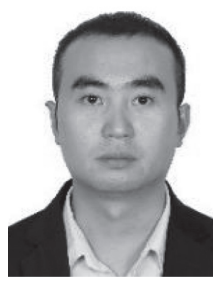

Zhongqiang Luo received the B.S. and M.S. degrees in communication engineering and pattern recognition and intelligent systems from Sichuan University of Science and Engineering, Zigong, China, in 2009 and 2012, respectively. He received the Ph.D. degree in communication and information systems from University of Electronic Science and Technology of China (UESTC), in 2016. Since 2017, he has been with the Sichuan University of Science and Engineering, where he is currently a lecturer. From December 2018-December 2019, he is a visiting scholar with Department of Computer Science and Electrical Engineering in University of Maryland Baltimore County (UMBC). His research interests include machine learning, blind source separation, signal processing for wireless communication system and intelligent signal processing.

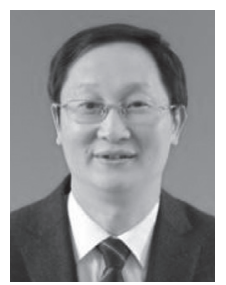

Xingzhong Xiong received the B.S. degrees in communication engineering from Sichuan University of Science and Engineering, Zigong, China, in 1996. He received the M.S and Ph.D. degrees in communication and information system from University of Electronic Science and Technology of China (UESTC), in 2006 and 2009, respectively. In 2012, he completed a research assignment from the Postdoctoral Station of Electronic Science and Technology at the UESTC. He is currently a professor at the School of Automation and Electronic Information, Sichuan University of Science and Engineering. His research interests include wireless and mobile communications technologies, intelligent signal processing, Internet of Things technologies, and very largescale integration (VLSI) designs.

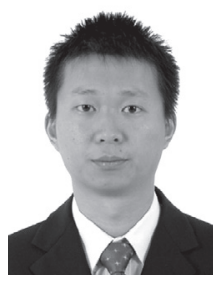

Kai Deng received the B.S., M.S. and Ph.D. degrees in communications and information systems from University of Electronic Science and Technology of China(UESTC), Chengdu, China, in 2002, 2005 and 2009, respectively. He is currently with Faculty of Intelligent Manufacturing, Yibin University, Yibin, China. His research interests mainly include signal processing in wireless communications. 